\title{
Reorganization of Visual Processing in Macular Degeneration Is Not Specific to the "Preferred Retinal Locus"
}

\author{
Daniel D. Dilks, ${ }^{1}$ Chris I. Baker, ${ }^{2}$ Eli Peli, ${ }^{3}$ and Nancy Kanwisher ${ }^{1}$ \\ ${ }^{1} \mathrm{McGovern}$ Institute for Brain Research, Massachusetts Institute of Technology, Cambridge, Massachusetts 02139, and ${ }^{2}$ Laboratory of Brain and Cognition, \\ National Institute of Mental Health-National Institutes of Health, Bethesda, Maryland 20892, and ${ }^{3}$ Schepens Eye Research Institute, Harvard Medical \\ School, Boston, Massachusetts 02114
}

Recent work has shown that foveal cortex, deprived of its normal bottom-up input as a result of macular degeneration (MD), begins responding to stimuli presented to a peripheral retinal location. However, these studies have only presented stimuli to the "preferred retinal location," or PRL, a spared part of the peripheral retina used by individuals with MD for fixating, face recognition, reading, and other visual tasks. Thus, previous research has not yet answered a question critical for understanding the mechanisms underlying this reorganization: Does formerly foveal cortex respond only to stimuli presented at the PRL, or does it also respond to other peripheral locations of similar eccentricity? If foveal cortex responds to stimuli at PRL because it is the long-term habitual use of this region as a functional fovea that drives the formerly foveal cortex to respond to stimuli presented at the PRL (the "use-dependent reorganization" hypothesis), then foveal cortex will not respond to stimuli presented at other locations. Alternatively, it may be that foveal cortex responds to any peripheral retinal input, independent of whether input at that retinal location has been chronically attended for months or years (the "use-independent reorganization" hypothesis). Using fMRI, we found clear activation of formerly foveal cortex to stimuli presented at either the PRL or an isoeccentric non-PRL location in two individuals with MD, supporting the use-independent reorganization hypothesis. This finding suggests that reorganization is driven by passive, not use-dependent mechanisms.

\section{Introduction}

Recent studies have demonstrated reorganization of visual processing in individuals with loss of foveal vision, and consequent loss of bottom-up input to "foveal" cortex, due to macular degeneration (MD). Specifically, in these individuals, stimuli presented to a peripheral retinal location elicited responses in the deprived cortex that would normally be responsive only to stimuli presented to the fovea (Baker et al., 2005, 2008; Masuda et al., 2008). However, these previous studies presented stimuli only to the "preferred retinal locus", or PRL, which is the part of the surviving peripheral retina chronically used as a "new fixation" center by people with MD. To test hypotheses about the mechanisms underlying reorganization, we ask here whether activation of formerly foveal cortex occurs only when stimuli are presented at the PRL, or whether it is also found for stimuli presented at other peripheral locations of similar eccentricity.

In individuals with MD, the PRL assumes the functional role of the former fovea: it is the part of the retina preferentially used

Received 0ct. 31, 2008; revised Jan. 9, 2009; accepted Jan. 27, 2009.

This work was supported by National Institutes of Health Grants EY016559 (N.K.) and EY005957 (E.P.), by Kirschstein National Research Service Award EY017507 (D.D.D.), by the National Institute of Mental Health Intramural Research Program (C.I.B.), and by Dr. Joseph Byrne and Nancy Byrne. We express our sincere thanks to the participants with macular degeneration and to Bradley Berk and Jonas Kubilius for help with data collection and analyses. We also thank the Athinoula A. Martinos Imaging Center at the McGovern Institute for Brain Research, Massachusetts Institute of Technology.

Correspondence should be addressed to Daniel D. Dilks, McGovern Institute for Brain Research, Massachusetts Institute of Technology, 46-4141, Cambridge, MA 02139. E-mail: dilks@mit.edu.

DOI:10.1523/JNEUROSCI.5258-08.2009

Copyright $\odot 2009$ Society for Neuroscience $\quad$ 0270-6474/09/292768-06\$15.00/0 for active, attention-demanding visual tasks such as reading and face recognition (Timberlake et al., 1987). Thus, according to one hypothesis (the "use-dependent reorganization" hypothesis), formerly foveal cortex responds in MD individuals when stimuli are presented at the PRL because the PRL has become the functional equivalent of the fovea. In other words, it is the long-term habitual use of this region as a functional fovea over months or years with persistent attentional focus to stimuli at this retinal location that drives the formerly foveal cortex to respond to stimuli presented at the PRL. Thus, the "use-dependent reorganization" hypothesis predicts that formerly foveal cortex will not be responsive to stimuli presented at an isoeccentric non-PRL location. However, the chronic fovea-like use of the PRL is not the only possible cause of the observed activation of formerly foveal cortex by stimuli presented at the PRL. According to the alternate "use-independent reorganization" hypothesis, formerly foveal cortex responds to peripheral stimuli simply because the foveal region gets no bottom-up input of its own, and peripheral regions do, leading the deprived foveal cortex to take any available input from cortex responding to peripheral stimuli. This hypothesis predicts that stimuli presented to both PRL and non-PRL locations will activate formerly foveal cortex.

To distinguish between these two accounts, we tested whether the deprived foveal cortex responds only to stimuli presented at the PRL (as predicted by the use-dependent reorganization hypothesis), or also to stimuli presented at an isoeccentric non-PRL location abutting the scotoma (as predicted by the useindependent reorganization hypothesis). Using functional magnetic resonance imaging (fMRI), we found robust activation of 


\begin{tabular}{|c|c|c|c|c|c|c|c|c|c|c|c|}
\hline \multirow[b]{3}{*}{ Participant } & \multirow[b]{3}{*}{ Gender } & \multirow{3}{*}{$\begin{array}{l}\text { Age of onset } \\
\text { (years) }\end{array}$} & \multirow{3}{*}{$\begin{array}{l}\text { Time since onset } \\
\text { (years) }\end{array}$} & \multirow{2}{*}{\multicolumn{2}{|c|}{ Visual acuity }} & \multirow[b]{3}{*}{ Diagnosis } & \multicolumn{4}{|c|}{ Fixation stability from Nidek } & \multirow{3}{*}{$\begin{array}{l}\text { Stimulus size } \\
\text { (degrees: } \mathrm{w} \times \mathrm{h} \text { ) }\end{array}$} \\
\hline & & & & & & & \multicolumn{2}{|l|}{ OD } & \multicolumn{2}{|l|}{ OS } & \\
\hline & & & & OD & OS & & $2^{\circ}(\%)$ & $4^{\circ}(\%)$ & $2^{\circ}(\%)$ & $4^{\circ}(\%)$ & \\
\hline MD8 & $\mathrm{F}$ & 24 & 26 & $20 / 100$ & $20 / 100$ & Stargardt's disease & 91 & 100 & 72 & 97 & $4 \times 4$ \\
\hline MD1 & M & 37 & 20 & $20 / 330^{*}$ & $20 / 330$ & Cone-rod dystrophy & 100 & 100 & N.A. & N.A. & $6 \times 6$ \\
\hline
\end{tabular}

An asterisk denotes the tested eye (no asterisk indicates that the participant was tested binocularly). OD, Right eye; OS, left eye; N.A., not available; w, width; h, height.

formerly foveal cortex to stimuli presented at either peripheral location in two individuals with bilateral MD. These findings are more consistent with the use-independent reorganization hypothesis than the use-dependent hypothesis, and suggest that reorganization is driven by passive, not use-dependent mechanisms.

\section{Materials and Methods}

Participants. We tested two individuals with MD: MD8, who had never been tested before, and MD1, who had been tested in a previous study (Baker et al., 2005) and had shown cortical reorganization to stimuli presented to the PRL. Both MD participants were carefully tested behaviorally to determine (1) visual field loss, including testing for any residual macular function, (2) location of the subject's PRL (Timberlake et al., 1986), and (3) fixation stability (Crossland et al., 2004). Participants were only selected for this study if they had extensive central scotomata, with complete bilateral loss of foveal function, and a single, stable PRL. For each MD individual we also tested a control participant (with full field vision) with visual stimuli presented to the same retinal locations as for their matched MD participant.

Visual field plotting. To document visual field loss, measurements were conducted using a custom computerized central perimetry system. A rear projection screen was used to present a uniform background of luminance $97 \mathrm{~cd} / \mathrm{m}^{2}$ and square target stimuli of luminance $0.28 \mathrm{~cd} / \mathrm{m}^{2}$ (Minolta LS-110 spot photometer). Each eye was tested separately. Participants were instructed to maintain fixation with their PRL on a fixation point at the center of the screen while a 19 $\mathrm{mm}\left(\sim 1^{\circ}\right)$ target was moved across the screen using a mouse. In an exploration phase (aided by the MP-1 measurements, discussed below), subjects were asked to report whenever the target disappeared. When the scotomatous areas were located, the target was placed inside the scotoma and moved from nonseeing to seeing regions (kinetic perimetry). The point of first seeing the target as reported by the participants was marked as the edge of the scotoma. Once the scotoma was mapped, targets were presented in random positions in the center of the scotoma in a search for any residual central vision. In MD8 static (seen/unseen) perimetry was also conducted in the MP-1, and it confirmed the location of the scotoma found with the custom perimeter described here.

Two-interval forced choice testing. We further tested for residual macular function by using a two-interval forced choice (2IFC) psychophysical test. MD participants fixated (with their PRL) on a fixation target. Each trial contained two temporal intervals, and a 1 cycle/degree horizontal Gabor patch ( $1^{\circ}$ in size, $90 \%$ contrast) was presented to either the fovea or PRL (50 trails each) in one of those intervals. The participant indicated which interval the Gabor patch was presented in, guessing when they were unsure. To avoid light scatter to functioning portions of the retina, the average luminance of the test patches was identical to the background.

Retinal imaging and perimetry. A Nidek MP-1 retinal microperimeter (Nidek Technologies) was used to map the location of the PRL, and to measure the stability of fixation at the PRL for MD participants. The retinal image tracker of the MP-1 recorded the subject's eye movement during $30 \mathrm{~s}$ fixation trials. This procedure provided a cluster of 750 samples of the location of the fixation cross on the retina ( 25 samples per second).
Functional imaging. Stimuli were grayscale photographs of objects (e.g., airplane, chair, watch) presented at the PRL, or an isoeccentric peripheral retinal location outside the patient's PRL (referred to as the non-PRL); and in the new participant (MD8) at the fovea. In the control participants, stimuli were presented at matched retinal locations. For a given MD participant, the images were the same size for all locations and were scaled for each MD participant so that the objects presented at PRL and non-PRL were large enough to be discriminated by the participant (Table 1).

MD8 (and her matched control) completed two runs, and MD1 (and his matched control) completed five runs of a simple blocked-design experiment. Participants viewed blocks of images (24 blocks of $16 \mathrm{~s}$ each for MD8 and matched control; and 16 blocks of $16 \mathrm{~s}$ each for MD1 and matched control) presented at each of the tested positions in each run. In each block, 20 images were presented for $500 \mathrm{~ms}$ each, with a $300 \mathrm{~ms}$ interstimulus interval. Participants performed a one-back task, responding via a button box every time they saw a consecutive repetition of the same object image.

MD8 was tested binocularly, while MD1 was tested monocularly. In MD1, the non-PRL location, if presented binocularly, would have been in the scotoma of the left eye, thus stimuli were presented to the right eye only. Each control participant viewed the stimuli with the same eye(s) as the corresponding MD participant. MD participants fixated on a cross $\left(2^{\circ}\right.$ by $\left.2^{\circ}\right)$ at the PRL location, whereas matched control participants fixated on a fixation cross at the foveal location. During scanning, eye movements were monitored in all participants, using an ISCAN ETL400 eye tracker.

Participants were scanned on a 3.0 T Siemens Trio scanner at the A. A. Martinos Imaging Center at the McGovern Institute, Massachusetts Institute of Technology. Functional images were acquired with a Siemens 12-channel phased-array head-coil and gradient echo single-shot echoplanar imaging sequence ( 22 slices, $2 \times 2 \times 2 \mathrm{~mm}, 0.2 \mathrm{~mm}$ interslice gap, $\mathrm{TR}=2 \mathrm{~s}, \mathrm{TE}=30 \mathrm{~ms}$ ). For all scans, slices were oriented approximately perpendicular to the calcarine sulcus. High-resolution anatomical images were also acquired for each participant for reconstruction of the cortical surface.

Data were analyzed using Freesurfer and FS-FAST software (http:// surfer.nmr.mgh.harvard.edu/). Before statistical analysis, images were motion corrected (Cox and Jesmanowicz, 1999), and smoothed ( $3 \mathrm{~mm}$ FWHM Gaussian kernel). Activations (stimulus conditions > fixation baseline) were visualized on the flattened cortical surface (Dale et al., 1999; Fischl et al., 1999) [for inflation and flattening procedures, see Baker et al. (2008), their Fig. 2]. To measure the magnitude of response at the occipital pole [the region of cortex responding to foveal stimulation (Dougherty et al., 2003)], a region of interest (ROI) was defined for all participants based on anatomical criteria. ROIs were drawn at the posterior end of the calcarine sulcus with a surface area in each hemisphere of $\sim 200 \mathrm{~mm}^{2}\left(168 \mathrm{~mm}^{2}\right.$ for MD8; $181 \mathrm{~mm}^{2}$ for MD1). Since the ROIs were defined based on the individual anatomy, there was some variation in the precise shape and size of the individual ROIs. These analyses were also performed on larger ROIs (surface area of $\sim 500 \mathrm{~mm}^{2}: 464 \mathrm{~mm}^{2}$ for MD8; $527 \mathrm{~mm}^{2}$ for MD1) producing nearly identical results. Activations in occipital pole ROIs to stimuli in different locations were compared with planned $t$ tests. 


\section{Results}

\section{Retinal data}

Both MD participants had extensive central scotomata and complete loss of foveal function as measured by perimetry. The 2IFC testing confirmed the perimetry findings, and showed that participants were at chance for stimuli presented in the center of the scotoma (former fovea), but with near perfect performance for stimuli presented at the PRL. Both MD participants had developed a clear, single stable PRL (mean $=99 \%$ of sample fixations were within $4^{\circ}$ ) (Table 1; supplemental Fig. 1, available at www.jneurosci.org as supplemental material). The distance of the PRL from the former fovea was $7.5^{\circ}$ for MD8 (both eyes), and $13.5^{\circ}$ for MD1 (right eye).

\section{fMRI data}

In both MD participants, visual stimulation at the PRL compared with the blank screen baseline produced responses at the occipital pole corresponding to the foveal confluence (white outlines show the occipital pole ROI in Figs. $1 A$ and $2 A$, column 2, labeled PRL). Crucially, visual stimulation at the non-PRL compared with baseline also elicited responses at the occipital pole (Figs. 1A, 2A, column 3, labeled nonPRL). As expected, visual activation was also observed in regions of cortex corresponding to PRL and non-PRL locations (arrows in Figs. $1 A$ and $2 A$; and column 4, labeled PRL-nonPRL) and in objectselective cortex. Presentation of stimuli at the fovea produced no activation at the occipital pole of MD8, confirming the total loss of foveal function. [The same was found for MD1, as reported in Baker et al. (2005) and in supplemental Fig. 2 (available at www.jneurosci.org as supplemental material).] In contrast, in control participants, stimulation at the PRL and non-PRL produced activation in respective peripheral retinotopic cortex and object-selective regions only; no activation was observed at the occipital pole (Fig. $3 A$ ). Stimulation of fovea produced robust occipital pole activation (supplemental Fig. 2, available at www.jneurosci.org as supplemental material).

To measure the magnitude of activation at the occipital pole, the average activation within an independently defined anatomical ROI was calculated for each MD and control participant. For both MD participants, there was significant activation in this occipital pole ROI to stimuli presented at the PRL compared with the fixation baseline (all $p$ values $<0.001$ ), and the non-PRL compared with the fixation baseline (all $p$ values $<0.01$ ) (Figs. $1 B$, $2 B)$. Further, no significant difference between stimuli presented at the PRL compared with the non-PRL was found (all $p$ values $>0.40$ ). No significant activation in the occipital pole ROI to stimuli presented at the fovea compared with fixation baseline the blocks.
MD8

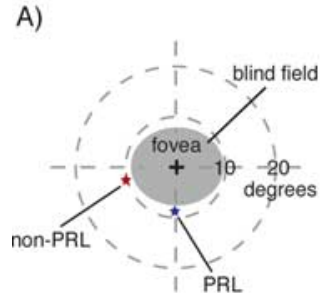

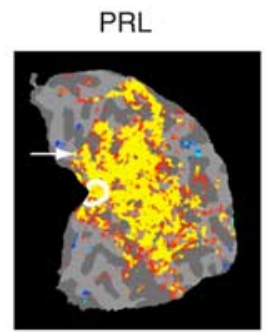

nonPRL
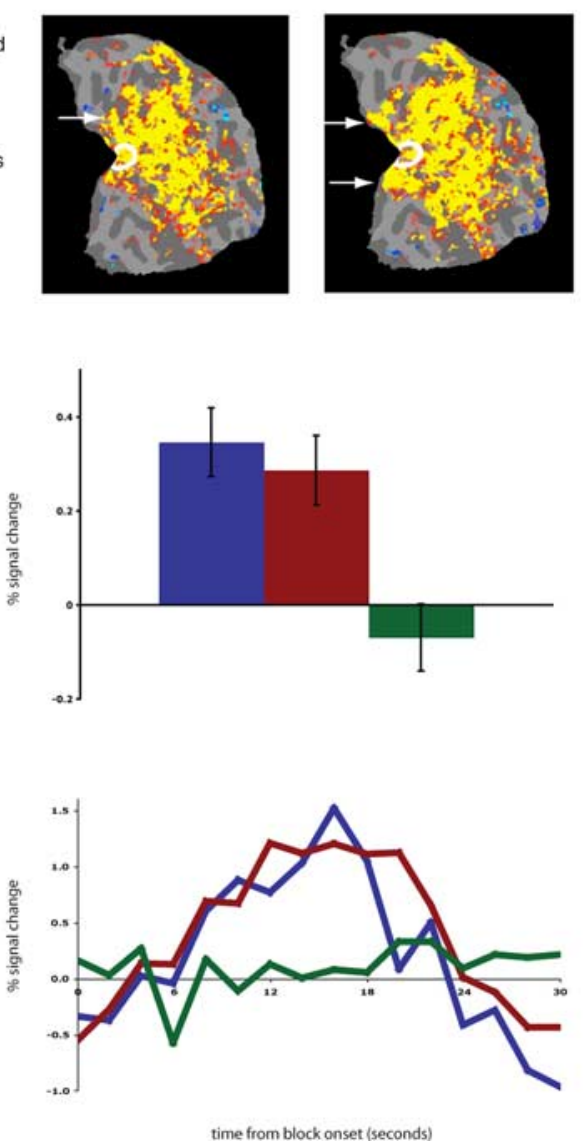

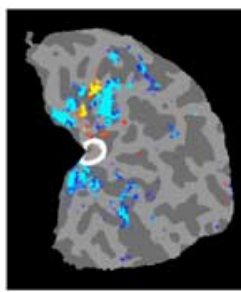

stmulus $>$ blank
$100^{6}$
blank $>$ stimulus
PRL-nonPRL

Figure 1. Reorganization of visual processing in MD8. A, Column 1, Schematic of the visual field in the left eye showing the large extent of the blind field (scotoma), the PRL (blue star), and the non-PRL (red star), labeled accordingly. MD8 was tested parametric maps on the flattened cortex showing activation at occipital pole ROI (white outlines show the occipital pole ROI). The activation maps are displayed on the flattened cortex and show activation in response to visual objects presented at the PR (column 2) and the non-PRL (column 3). In MD8, the non-PRL was located in the left visual field, and thus the data are shown for the right hemisphere only. In each case, activation was observed not only in parts of cortex corresponding to the retinal location parametric map on the flattened cortex showing activation for the contrast of PRL versus non-PRL. This activation map shows the expected activation in response to visual objects presented at the PRL (yellow), and the non-PRL (blue), but not at the occipital (white outlines on the statistical maps). Stimuli presented at the PRL (blue bar) and the non-PRL (red bar) elicited strong responses in the occipital pole ROI, while stimuli presented at the fovea (green bar) elicited no response. $\boldsymbol{C}$, Average time course of activation in the occipital pole ROI. There is a strong increase in activation relative to the fixation baseline for stimuli presented at the PRL (blue line) and the non-PRL (red line), but no response to stimuli presented at the fovea (green line) over the course of 
MD1

A)

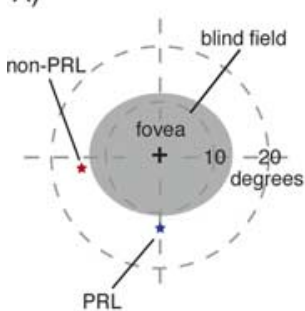

B)
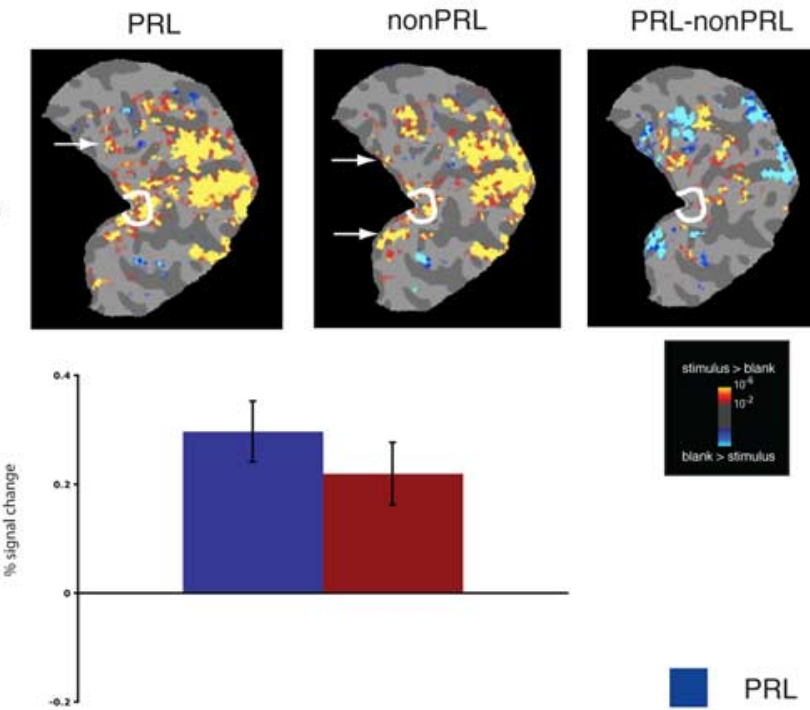

C)

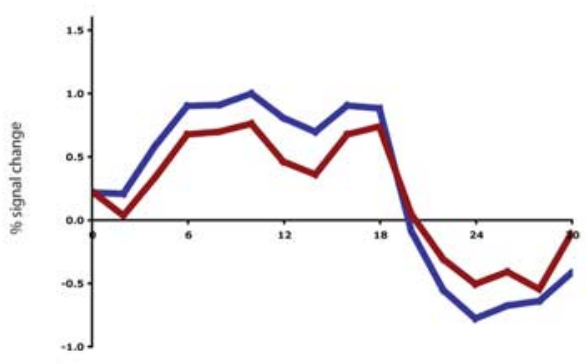

time from block onset (seconds)

Figure 2. Reorganization of visual processing in MD1. A, Column 1, Schematic of the visual field in the right eye showing the large extent of the blind field (scotoma), the PRL (blue star), and the non-PRL (red star), labeled accordingly. MD1's right eye only was tested because the non-PRL location, if presented binocularly, would have been in the scotoma of the left eye. $A$, Columns 2 and 3, Statistical parametric maps on the flattened cortex showing activation at occipital pole ROI (white outlines show the occipital pole ROI). The activation maps are displayed on the flattened cortex and show activation in response to visual objects presented at the PRL (column 2) and the non-PRL (column 3). In MD1, the non-PRL was located in the left visual field, and thus the data are shown for the right hemisphere only. In each case, activation was observed not only in parts of cortex corresponding to the retinal location of the PRL and non-PRL (arrows), but also in the occipital pole corresponding to the foveal confluence. $A$, Column 4, Statistical parametric map on the flattened cortex showing activation for the contrast of PRL versus non-PRL. This activation map shows the expected activation in response to visual objects presented at the PRL (yellow) and the non-PRL (blue), but not at the occipital pole. $\boldsymbol{B}$, Bar chart showing percentage signal change (from fixation baseline) in the independently defined occipital pole ROI (white outlines on the statistical maps). Stimuli presented at the PRL (blue bar) and the non-PRL (red bar) elicited strong responses in the occipital pole ROI. C, Average time course of activation in the occipital pole ROl. There is a strong increase in activation relative to the fixation baseline for stimuli presented at the PRL (blue line) and the non-PRL (red line) over the course of the blocks.

MD1 in the study by Baker et al. (2005)]. Again, the opposite pattern was observed in the matched control participants: little or no change in activation relative to the fixation baseline for peripheral stimuli but strong increases in activity for stimuli presented at the fovea (Fig. 3C).

Thus, the three separate analyses described above provide converging evidence that following the loss of foveal input, the deprived region of cortex that would normally be responsive only to foveal visual stimuli responds to stimuli presented at both PRL and non-PRL locations. Furthermore, both MD participants were accurate at maintaining fixation during scanning (supplemental Fig. 3, available at www.jneurosci.org as supplemental material). Thus, the activation of foveal cortex by stimuli at nonPRL cannot be due to fixation of these stimuli with the PRL.

\section{Discussion}

In two individuals with MD, we found activation of formerly foveal cortex by visual stimuli presented at either of two peripheral locations, the PRL and a non-PRL location of similar eccentricity. This finding supports the proposed "use-independent reorganization" hypothesis, not the "usedependent reorganization" hypothesis. According to the use-independent reorganization hypothesis, deprived foveal cortex comes to respond to peripheral stimuli simply because the foveal region gets no bottom-up input of its own, and peripheral regions do, leading the deprived foveal cortex to take input from any cortex responsive to the peripheral stimuli regardless of the behavioral significance of the PRL.

Note that the use-dependent reorganization hypothesis concerns long-term use of a PRL before the testing session, not simply fovea-like attention to that retinal location at test. It is a different (and important) question whether activation of foveal cortex by peripheral stimuli occurs only when those stimuli are attended at test. Current evidence indicates that attention enhances activation in foveal cortex in MD individuals (Baker et al., 2008; Masuda et al., 2008), but it is not clear that it is required. If attention is required for the effect, this could be mediated directly by feedback or by feedback that acts by modulating horizontal connections (Gilbert and Sigman, 2007).

Our finding that reorganization of visual processing is not specific to retinal locations that have become the functional equivalent of the fovea is consistent with several previous findings demonstrating dissociation between reorganization and the adoption of a PRL. First, reorganization of primary visual cortex (V1) has been shown in adult animals following discrete retinal lesions (Kaas et al., 1990; Heinen and Skavenski, 1991; Chino et al., 1992; Gilbert and Wiesel, 1992; Darian-Smith and Gilbert, 1995; Schmid et al., 1996; Calford et al., 2000; Keck et al., 2008). In most of these studies, the lesions are in the peripheral retina, not the fovea; these animals do not adopt a PRL, yet reorganization occurs (but see Smirnakis et al., 2005). Specifically, deprived V1 neurons (i.e., as a result of retinal lesioning), begin responding to stimuli that normally activate adjacent cortex only. Second, V1 reorganization has also been demonstrated in the case of a stroke patient with damage affecting the peripheral visual field only. This patient also did not adopt a PRL, yet the deprived visual cortex representing the upper left visual field began responding to input from cortex representing the lower left visual field (Dilks et al., 2007). Third, some individuals with MD, who have adopted a PRL, do not show evidence for large-scale reorganization (Baker et al., 2008). Thus, reorganization can occur in the absence of a 
new functional fovea, and can fail to occur in the presence of a new functional fovea.

While our question in this study was whether foveal cortex is responsive to stimuli presented away from the PRL (i.e., non-PRL stimuli), the further question of whether stimuli at the PRL might activate foveal cortex more strongly than stimuli presented at a non-PRL location (which seems to be the trend in the two individuals reported in this paper) is an interesting one. However, any such difference might be difficult to interpret given that participants are more adept at attending to PRL than non-PRL locations, and could simply reflect attentional differences.

In conclusion, we have demonstrated that reorganization of visual processing in individuals with MD is not specific to the retinal location that has become the functional equivalent of the fovea, the PRL, suggesting that reorganization in driven by passive, not use-dependent mechanisms.

Note added in proof. Schumacher et al. (2008) have just published a paper that finds activation of foveal cortex only for stimuli at the PRL, not stimuli presented elsewhere. The reason for this conflicting result is not entirely clear.

\section{References}

Baker CI, Peli E, Knouf N, Kanwisher NG (2005) Reorganization of visual processing in macular degeneration. J Neurosci 25:614-618.

Baker CI, Dilks DD, Peli E, Kanwisher N (2008) Reorganization of visual processing in macular degeneration: replication and clues about the role of foveal loss. Vision Res 48:19101919.

Calford MB, Wang C, Taglianetti V, Waleszczyk WJ, Burke W, Dreher B (2000) Plasticity in adult cat visual cortex (area 17) following circumscribed monocular lesions of all retinal layers. J Physiol 524:587-602.

Chino YM, Kaas JH, Smith EL 3rd, Langston AL, Cheng H (1992) Rapid reorganization of cortical maps in adult cats following restricted deafferentation in retina. Vision Res 32:789-796.

Cox RW, Jesmanowicz A (1999) Real-time 3D image registration for functional MRI. Magn Reson Med 42:1014-1018.

Crossland MD, Culham LE, Rubin GS (2004) Fixation stability and reading speed in patients with newly developed macular disease. Ophthalmic Physiol Opt 24:327-333.

Dale AM, Fischl B, Sereno MI (1999) Cortical surface-based analysis. I. Segmentation and surface reconstruction. Neuroimage 9:179194.

Darian-Smith C, Gilbert CD (1995) Topographic reorganization in the striate cortex of the adult cat and monkey is cortically mediated. J Neurosci 15:1631-1647.

Dilks DD, Serences JT, Rosenau BJ, Yantis S, McCloskey M (2007) Human adult cortical reorganization and consequent visual distortion. J Neurosci 27:9585-9594.

\section{MD8 Control}

A)

B)

\section{MD1 Control}

A) presented at the fovea.
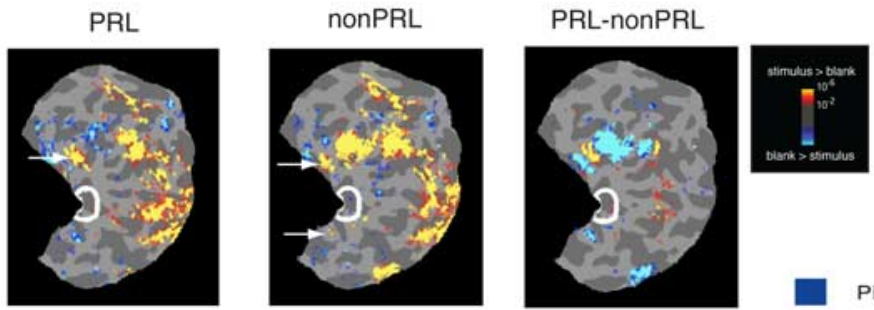

C)

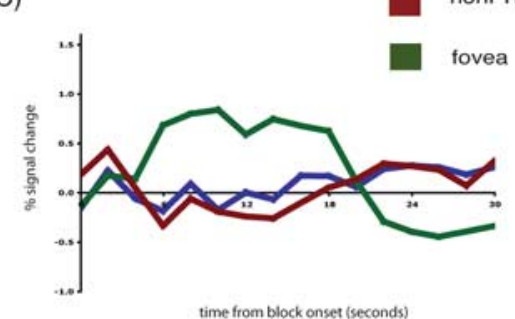

time from block onset (seconds)
PRL

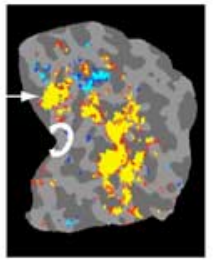

B)

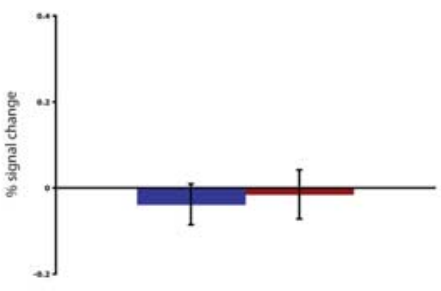

nONPRL

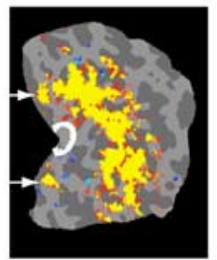

C)

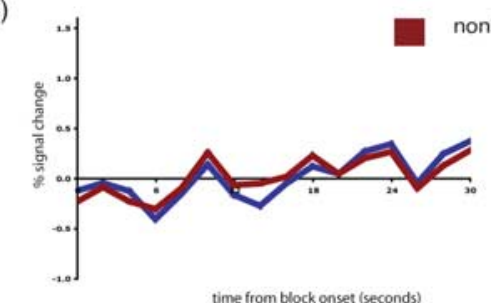

PRL-nOnPRL
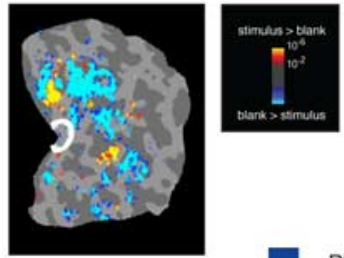

PRL nONPRL
Figure 3. Lack of reorganization of visual processing in matched controls, labeled accordingly. A, Columns 1 and 2, Statistical parametric maps on the flattened cortex showing no activation at the occipital pole (white outlines show the occipital pole ROIs). The activation maps are displayed on the flattened cortex and show activation in response to visual objects presented at the PRL (column 2) and the non-PRL (column 3). In each case, activation was observed only in parts of cortex corresponding to the retinal location of the PRL and non-PRL (arrows), not at the occipital pole corresponding to the foveal confluence. $\boldsymbol{A}$, Column 3, Statistical parametric map on the flattened cortex showing activation for the contrast of PRL versus non-PRL. This activation map shows the expected activation in response to visual objects presented at the PRL (yellow) and the non-PRL (blue). $\boldsymbol{B}$, Bar chart showing percentage signal change (from fixation baseline) in the independently defined occipital pole ROI (white outlines on the statistical maps). Stimuli presented at the PRL (blue bar) and the non-PRL (red bar) elicited no discernable responses above baseline in the occipital pole ROI. In contrast, for MD8 Control, stimuli presented at the fovea elicited a strong response (green bar). $\boldsymbol{C}$, Average time course of activation in the occipital pole ROI. There is no activation relative to the fixation baseline for stimuli presented at the PRL (blue line) and the non-PRL (red line) over the course of the blocks, and the expected activation for stimuli

Dougherty RF, Koch VM, Brewer AA, Fischer B, Modersitzki J, Wandell BA (2003) Visual field representations and locations of visual areas $\mathrm{V} 1 / 2 / 3$ in human visual cortex. J Vis 3:586-598.

Fischl B, Sereno MI, Dale AM (1999) Cortical surface-based analysis. II: Inflation, flattening, and a surface-based coordinate system. Neuroimage 9:195-207.

Gilbert CD, Sigman M (2007) Brain states: top-down influences in sensory processing. Neuron 54:677-696.

Gilbert CD, Wiesel TN (1992) Receptive field dynamics in adult primary visual cortex. Nature 356:150-152.

Heinen SJ, Skavenski AA (1991) Recovery of visual responses in foveal V1 
neurons following bilateral foveal lesions in adult monkey. Exp Brain Res 83:670-674.

Kaas JH, Krubitzer LA, Chino YM, Langston AL, Polley EH, Blair N (1990) Reorganization of retinotopic cortical maps in adult mammals after lesions of the retina. Science 248:229-231.

Keck T, Mrsic-Flogel TD, Vaz Afonso M, Eysel UT, Bonhoeffer T, Hübener M (2008) Massive restructuring of neuronal circuits during functional reorganization of adult visual cortex. Nat Neurosci 11:1162-1167.

Masuda Y, Dumoulin SO, Nakadomari S, Wandell BA (2008) V1 projection zone signals in human macular degeneration depend on task, not stimulus. Cereb Cortex 18:2483-2493.

Schmid LM, Rosa MG, Calford MB, Ambler JS (1996) Visuotopic reorganization in the primary visual cortex of adult cats following monocular and binocular retinal lesions. Cereb Cortex 6:388-405.
Schumacher EH, Jacko JA, Primo SA, Main KL, Moloney KP, Kinzel EN, Ginn J (2008) Reorganization of visual processing is related to eccentric viewing in patients with macular degeneration. Restor Neurol Neurosci 26:391-402.

Smirnakis SM, Brewer AA, Schmid MC, Tolias AS, Schüz A, Augath M, Inhoffen W, Wandell BA, Logothetis NK (2005) Lack of long-term cortical reorganization after macaque retinal lesions. Nature 435:300307.

Timberlake GT, Mainster MA, Peli E, Augliere RA, Essock EA, Arend LE (1986) Reading with a macular scotoma. I. Retinal location of scotoma and fixation area. Invest Ophthalmol Vis Sci 27:1137-1147.

Timberlake GT, Peli E, Essock EA, Augliere RA (1987) Reading with a macular scotoma. II. Retinal locus for scanning text. Invest Ophthalmol Vis Sci 28:1268-1274. 